International Journal of Pure and Applied Mathematics

Volume 112 No. 4 2017, 673-682

ISSN: 1311-8080 (printed version); ISSN: 1314-3395 (on-line version)

url: http://www.ijpam.eu

doi: $10.12732 /$ ijpam.v112i4.2

ijpam.eu

\title{
MONOTONE ITERATIVE TECHNIQUE FOR FINITE SYSTEM OF FRACTIONAL DIFFERENCE EQUATIONS
}

\author{
D.N. Purnima ${ }^{1}$, G.V.S.R. Deekshitulu ${ }^{2} \S$ \\ ${ }^{1}$ Department of Mathematics, RIET \\ Rajahmundry, INDIA \\ Department of Mathematics \\ JNT University Kakinada \\ Kakinada, Andhra Pradesh, INDIA
}

\begin{abstract}
In this paper, we consider non-linear fractional finite difference system and establish the existence of solutions using monotone iterative technique.
\end{abstract}

AMS Subject Classification: 39A10, 39A99

Key Words: fractional order, finite system, difference inequality, monotone iterative technique

\section{Introduction}

Fractional calculus gained importance during the past three decades due to its applicability in diverse fields of science and engineering. The notions of fractional calculus may be traced back to the works of Euler, but the idea of fractional difference is very recent. G.V.S.R. Deekshitulu and J. Jagan Mohan [2] modified the definition of fractional difference given by Atsushi Nagai [11] and discussed some basic inequalities, comparison theorems and qualitative properties of the solutions of fractional difference equations $[2,3,4,5,6]$.

\footnotetext{
Received: $\quad$ April 4, 2016

Revised: November 12, 2016

Published: February 13, 2017

(c) 2017 Academic Publications, Ltd. url: www.acadpubl.eu

${ }^{\S}$ Correspondence author
} 
Being an important tool in the study of existence, uniqueness, boundedness, stability and other qualitative properties of solutions of finite systems of differential equations and difference equations, comparison theorems and their applications have attracted great interests of many mathematicians. The investigation of scalar fractional order difference inequalities was initiated by G.V.S.R. Deekshitulu and J. Jagan Mohan [2].

In this paper, we use monotone iterative technique for finite system of fractional difference equations and derive the convergence of monotone sequences to upper and lower solutions. Then using these upper and lower solutions, existence of solutions to finite system of fractional difference inequalities to obtain more general results.

\section{Preliminaries}

In this section, we introduce some basic definitions and results concerning nabla discrete fractional calculus.

Definition 2.1. The backward difference operator $\Delta_{-n}$ is defined as $\Delta_{-n}=\varepsilon^{-1}(1-B)$ where $B f_{n}=f_{n-1}$ is standard backward shift operator and $\varepsilon$ is interval length.

Henry L Gray and Nien fan Zhang gave a definition of the fractional difference as follows:

Definition 2.2. For any complex number $\alpha$ and $f$ defined over the integer set $\{a-p, a-p+1, \ldots . n\}$, the $\alpha^{t h}$ order difference of $f(n)$ over $\{a, a+1, \ldots . n\}$ is defined by

$$
\nabla^{\alpha} f(n)=\frac{\nabla^{p}}{\Gamma(p-\alpha)} \sum_{k=0}^{n-a} \frac{\Gamma(k+p-\alpha)}{\Gamma(k+1)} f(n-k) .
$$

Later, Hirota took the first $n$ terms of Taylor series of $\Delta_{-n}^{\alpha}=\varepsilon^{-\alpha}(1-B)^{\alpha}$ and gave the following definition.

Definition 2.3. Let $\alpha \in \mathbb{R}$. Then difference operator of order $\alpha$ is defined by

$$
\Delta_{-n}^{\alpha} u_{n}= \begin{cases}\varepsilon^{-\alpha} \sum_{j=0}^{n-1}\left(\begin{array}{c}
\alpha \\
j
\end{array}\right)(-1)^{j} u_{n-j}, & \alpha \neq 1,2, \ldots \\
\varepsilon^{-m} \sum_{j=0}^{m}\left(\begin{array}{c}
m \\
j
\end{array}\right)(-1)^{j} u_{n-j}, & \alpha=m \in \mathbb{Z}_{>0} .\end{cases}
$$


Here $\left(\begin{array}{l}a \\ n\end{array}\right),(a \in \mathbb{R}, n \in \mathbb{Z})$ stands for a binomial coefficient defined by

$$
\left(\begin{array}{l}
a \\
n
\end{array}\right)= \begin{cases}\frac{\Gamma(a+1)}{\Gamma(a-n+1) \Gamma(n+1)} & \mathrm{n}>0 \\
1 & \mathrm{n}=0 \\
0 & \mathrm{n}<0\end{cases}
$$

In 2002, Atsushi Nagai [1] introduced another definition of fractional difference which is a slight modification of Hirota's fractional difference operator.

Definition 2.4. Let $\alpha \in \mathbb{R}$ and $m$ be an integer such that $m-1<\alpha \leq m$. The difference operator $\Delta_{*,-n}$ of order $\alpha$ is defined as

$$
\Delta_{*,-n}^{\alpha} u_{n}=\Delta_{-n}^{\alpha-m} \Delta_{-n}^{m} u_{n}=\varepsilon^{m-\alpha} \sum_{j=0}^{n-1}\left(\begin{array}{c}
\alpha-m \\
j
\end{array}\right)(-1)^{j} \Delta_{-(n-j)}^{m} u_{n-j} .
$$

G.V.S.R.Deekshitulu and J.Jagan Mohan [2] rearranged the terms in Atsushi Nagai's [11] definition for $0<\alpha<1$ in such a way that the expression for $\nabla^{\alpha}$ does not involve any difference operator and the term $(-1)^{j}$ inside the summation index as follows.

Definition 2.5. The fractional sum operator of order $\alpha$ is defined as

$$
\nabla^{-\alpha} u(n)=\sum_{j=0}^{n-1}\left(\begin{array}{c}
j+\alpha-1 \\
j
\end{array}\right) u(n-j)=\sum_{j=1}^{n}\left(\begin{array}{c}
n-j+\alpha-1 \\
n-j
\end{array}\right) u(j) .
$$

Definition 2.6. The Caputo type fractional difference operator of order $\alpha$ is defined as

$$
\nabla^{\alpha} u(n)=\nabla^{\alpha-1}[\nabla u(n)]=\sum_{j=0}^{n-1}\left(\begin{array}{c}
j-\alpha \\
j
\end{array}\right) \nabla u(n-j) .
$$

Corollary 1. The equivalent form of (2.6) is

$$
\nabla^{\alpha} u(n)=u(n)-\left(\begin{array}{c}
n-\alpha-1 \\
n-1
\end{array}\right) u(0)-\alpha \sum_{j=1}^{n-1} \frac{1}{j-\alpha}\left(\begin{array}{c}
j-\alpha \\
j
\end{array}\right) u(j) .
$$

Theorem 2.1. (Discrete Langenhop Inequality) Let $y(n), a(n)$ and $b(n)$ be any three non negative functions defined for $n \in \mathbb{N}_{0}^{+}$. If for $n, k \in \mathbb{N}_{0}^{+}$such that $k \leq n$ the following inequality be satisfied

$$
y(n) \geq y(k)-b(n) \sum_{j=k+1}^{n}[a(j) y(j)]
$$


where $y(n)$ is not necessarily non negative. Then, for all $n, k \in \mathbb{N}_{0}^{+}$such that $k \leq n$

$$
y(n) \geq y(k) \prod_{j=k+1}^{n}[1+b(n) a(j)]^{-1} .
$$

Theorem 2.2. Let $u(n)$ and $v(n)$ be non-negative function defined for $n \in N_{0}^{+}$such that $u(0)=v(0)$.For $0<\alpha<1$, if $u(n) \leq v(n)$ for $n \in N_{1}^{+}$then $\nabla^{-\alpha} u(n) \leq \nabla^{-\alpha} v(n)$.

\section{Main Results}

In this section, we consider the following finite system of non linear fractional difference equations of order $\alpha, 0<\alpha<1$

$$
\nabla^{\alpha} u(n+1)=f(n, u(n)), u(0)=u_{0} .
$$

where $u$ and $f$ are $n$-vectors with components $u_{i}(n): \mathbb{N}_{0}^{+} \longrightarrow \mathbb{R}$ and $f_{i}$ : $\mathbb{N}_{0}^{+} \times \mathbb{R} \longrightarrow \mathbb{R}, 1 \leq i \leq n$. We first establish some basic vectorial inequalities. Vectorial inequalities mean that the same inequalities hold between the corresponding components. We need the following properties in this study.

Definition 3.1. A function $f_{i}=f_{i}\left(u_{1}, u_{2}\right)$ is said to be quasi monotone non-decreasing (non-increasing) if for any fixed $u_{i}(i=1,2) f_{i}$ is non-decreasing (non-increasing) in $u_{j}$ for $j \neq i$.

Definition 3.2. A function $f \in C\left[\mathbb{N}_{0}^{+} \times \mathbb{R}^{n}, \mathbb{R}^{n}\right]$ is said to be quasi monotone non decreasing (non increasing) if , for each $i$ such that $1 \leq i \leq n, u \leq v$ and $u_{i}=v_{i}, f_{i}(t, u) \leq f_{i}(t, v) \quad\left(f_{i}(t, u) \geq f_{i}(t, v)\right)$.

It means that if for all $i, f_{i}$ are quasi monotone non-decreasing( quasi monotone non increasing), then $f$ is said to be quasi monotone non decreasing quasi monotone non increasing).

Definition 3.3. A function $v(n): \mathbb{N}_{0}^{+} \rightarrow \mathbb{R}^{n}$ is said to be a lower solution of $(3.1)$ if

$$
\nabla^{\alpha} v(n+1) \leq f(n, v(n)), \quad v_{0} \leq u_{0} .
$$

Similarly a function $w(n): \mathbb{N}_{0}^{+} \rightarrow \mathbb{R}^{n}$ is said to be an upper solution of (3.1) if

$$
\nabla^{\alpha} w(n+1) \geq f(n, w(n)), \quad w_{0} \geq u_{0} .
$$

Theorem 3.1. Let the function $f(n, v(n))$ be quasi monotone non-decreasing and $v(n)$ and $w(n)$ be lower and upper solutions of (3.1) defined on $\mathbb{N}_{0}^{+}$such that $\mathbf{v}(0) \leq \mathbf{w}(0)$. Then for all $n \in \mathbb{N}_{0}^{+}, v(n) \leq w(n)$. 
Proof. Suppose there exists a $k \in N_{0}^{+}$such that $v(k) \leq w(k)$ and $v(k+1)>$ $w(k+1)$.

Since $0<\alpha<1 \leq j$ and $\left(\begin{array}{c}j-\alpha \\ j\end{array}\right)>0$, we have

$$
\begin{aligned}
\nabla^{\alpha} v(k+1) & =v(k+1)-\left(\begin{array}{c}
k-\alpha \\
k
\end{array}\right) v(0)-\alpha \sum_{j=1}^{k} \frac{1}{j-\alpha}\left(\begin{array}{c}
j-\alpha \\
j
\end{array}\right) v(k+1-j) \\
& >w(k+1)-\left(\begin{array}{c}
k-\alpha \\
k
\end{array}\right) w(0)-\alpha \sum_{j=1}^{k} \frac{1}{j-\alpha}\left(\begin{array}{c}
j-\alpha \\
j
\end{array}\right) w(k+1-j) \\
& =\nabla^{\alpha} w(k+1) .
\end{aligned}
$$

Or $f(k, w(k)) \leq \nabla^{\alpha} w(k+1)<\nabla^{\alpha} v(k+1) \leq f(k, v(k))$

i.e., $f(k, w(k))<f(k, v(k))$. This is a contradiction to the quasi monotone property of $f(n, u(n))$. Hence the proof.

Further, if $u(n)$ is a solution of (3.1), then the by repeatedly applying the above theorem, we obtain

$$
v(n) \leq u(n) \leq w(n)
$$

Theorem 3.2. Suppose that $\nabla^{\alpha} m_{n+1} \leq-M m_{n}$ for $M>0$ and $m_{0} \leq 0$, then $m_{n} \leq 0$.

Proof. If it is false, then there exists a $k \in \mathbb{N}_{0}^{+}$such that $m_{k}>0$ and $m_{n} \leq 0$ for $n<k$. Consider

$$
\begin{gathered}
\quad \nabla^{\alpha} m_{k}=m_{k}-\left(\begin{array}{c}
k-1-\alpha \\
k-1
\end{array}\right) m_{0}+\sum_{j=1}^{k-1}\left(\begin{array}{c}
j-\alpha-1 \\
j
\end{array}\right) m_{k-j} \leq-M m_{k} \\
\text { or }(M+1) m_{k}+\sum_{j=1}^{k-1}\left(\begin{array}{c}
j-\alpha-1 \\
j
\end{array}\right) m_{k-j}-\left(\begin{array}{c}
k-1-\alpha \\
k-1
\end{array}\right) m_{0} \leq 0
\end{gathered}
$$

Since $m_{k}>0, m_{n} \leq 0$ for $n=0,1,2, \ldots, k-1,\left(\begin{array}{c}j-\alpha \\ j\end{array}\right)>0$ and $\left(\begin{array}{c}j-\alpha-1 \\ j\end{array}\right)<0$ implies that it is a contradiction. Hence $m_{n} \leq 0$ for $n \in \mathbb{N}_{0}^{+}$.

\section{Monotone iterative technique:}

Now we shall apply a general theory of monotone iterative technique for finite system of fractional difference equations of order $\alpha(0<\alpha<1$. ) We need 
the following notions.

For each $i, 1 \leq i \leq n$, let $p_{i}, q_{i}$ be two non-negative integers such that $p_{i}+q_{i}=n-1$ so that we can split the vector $u$ into $u=\left(u_{i},[u]_{p i},[u]_{q i}\right)$. Then the system (3.1)can be written as

$$
\left.\nabla^{\alpha} u_{i+1}=f_{i}\left(n, u_{i},[u]_{p i},[u]_{q i}\right)\right), u(0)=u_{0},
$$

for $1 \leq i \leq n$.

Definition 3.4. Let $v, w \in C^{1}\left[N_{0}^{+}, R^{n}\right]$, then $v, w$ are said to be coupled lower and upper quasi solutions of (3.4), if

$$
\begin{aligned}
\nabla^{\alpha} v_{i+1} & \left.\leq f_{i}\left(n, v_{i},[v]_{p i},[w]_{q i}\right)\right), v(0) \leq u_{0} \\
\nabla^{\alpha} w_{i+1} & \left.\geq f_{i}\left(n, w_{i},[w]_{p i},[v]_{q i}\right)\right), w(0) \geq u_{0}
\end{aligned}
$$

$v$ and $w$ are said to be coupled quasi solutions of (3.4) if

$$
\begin{aligned}
\nabla^{\alpha} v_{i+1} & \left.=f_{i}\left(n, v_{i},[v]_{p i},[w]_{q i}\right)\right), v(0)=u_{0} \\
\nabla^{\alpha} w_{i+1} & \left.=f_{i}\left(n, w_{i},[w]_{p i},[v]_{q i}\right)\right), w(0)=u_{0} .
\end{aligned}
$$

Definition 3.5. A function $f \in C\left[N_{0}^{+} \times R^{n}, R^{n}\right]$ is said to possess a mixed quasi monotone property (mqmp), if for each $i, f_{i}\left(n, u_{i},[u]_{p i},[u]_{q i}\right)$ is monotone non-decreasing in $[u]_{p i}$ components and monotone non-increasing in $[u]_{q i}$ components.

In particular $f(n, u)$ is said to possess non-decreasing property if $f_{i}(n, u)$ is non-decreasing in $u_{1}, u_{2}, \ldots, u_{n}$ for all fixed $n \in N_{0}^{+}$.

Theorem 3.3. Let the function $f(n, u)$ possess mixed monotonic property. Further, let there exists functions $v(n)$ and $w(n)$ defined on $N_{0}^{+}$such that

$$
\begin{aligned}
\nabla^{\alpha} v_{p}(n+1) & \leq f_{p}(n, v(n)) \\
\nabla^{\alpha} v_{q}(n+1) & \geq f_{q}(n, v(n)) \\
\nabla^{\alpha} w_{p}(n+1) & \geq f_{p}(n, w(n)) \\
\nabla^{\alpha} w_{q}(n+1) & \leq f_{q}(n, w(n)) .
\end{aligned}
$$

Then for all $n \in N_{0}^{+}, v_{p}(n) \leq w_{p}(n), v_{q}(n) \geq w_{q}(n)$ provided $v_{p}(0) \leq w_{p}(0), v_{q}(0)$ $\geq w_{q}(0)$.

Proof. Define a function $z(n)$ as follows:

$$
z_{p}(n)=w_{p}(n)-v_{p}(n) \text { and } z_{q}(n)=v_{q}(n)-w_{q}(n)
$$


By using principle of mathematical induction we shall show that $z_{i}(n) \geq 0$ for all $n \in N_{0}^{+}$. We have, $v_{p}(0) \leq w_{p}(0), v_{q}(0) \geq w_{q}(0)$ i.e., $z_{p}(0) \geq 0, z_{q}(0) \geq 0$. Let $v_{p}(m) \leq w_{p}(m), v_{q}(m) \geq w_{q}(m)$ for some fixed $m \in N_{0}^{+}$or $z_{p}(m) \geq 0$, $z_{q}(m) \geq 0$. Now consider

$$
\begin{aligned}
v_{p}(m+1) \leq & \left(\begin{array}{c}
m-\alpha \\
m
\end{array}\right) v_{p}(0)+\alpha \sum_{j=1}^{m} \frac{1}{j-\alpha}\left(\begin{array}{c}
j-\alpha \\
j
\end{array}\right) v_{p}(m+1-j) \\
& +F_{p}(m, v(m)) \\
\leq & \left(\begin{array}{c}
m-\alpha \\
m
\end{array}\right) w_{p}(0)+\alpha \sum_{j=1}^{m} \frac{1}{j-\alpha}\left(\begin{array}{c}
j-\alpha \\
j
\end{array}\right) w_{p}(m+1-j) \\
& +F_{p}(m, w(m)) \\
\leq & w_{p}(m+1) . \\
v_{q}(m+1) \geq & \left(\begin{array}{c}
m-\alpha \\
m
\end{array}\right) v_{q}(0)+\alpha \sum_{j=1}^{m} \frac{1}{j-\alpha}\left(\begin{array}{c}
j-\alpha \\
j
\end{array}\right) v_{q}(m+1-j) \\
& +F_{q}(m, v(m)) \\
\geq & \left(\begin{array}{c}
m-\alpha \\
m
\end{array}\right) w_{q}(0)+\alpha \sum_{j=1}^{m} \frac{1}{j-\alpha}\left(\begin{array}{c}
j-\alpha \\
j
\end{array}\right) w_{q}(m+1-j) \\
& +F_{q}(m, w(m)) \\
\geq & w_{q}(m+1) .
\end{aligned}
$$

By principle of mathematical induction, $z_{i}(n) \geq 0$ for all $n \in N_{0}^{+}$i.e., $z(n) \geq 0$ for all $n \in N_{0}^{+}$implies $v_{p}(n) \leq w_{p}(n), v_{q}(n) \geq w_{q}(n)$. Hence the proof.

Theorem 3.4. Let the assumptions of the Theorem 3.2 hold good and $u(n)$ is the solution of (3.1). If $v(0)=w(0)=u(0)=u_{0}$, then for all $n \in \mathbb{N}_{0}^{+}$,

$$
v_{p}(n) \leq u_{p}(n) \leq w_{p}(n) \quad \text { and } \quad w_{q}(n) \leq u_{q}(n) \leq v_{q}(n)
$$

If $u$ is a solution of (3.4), then the result follows from Theorems (3.1) and $(3.2)$.

Theorem 3.5. Let $f \in C\left[N_{0}^{+} \times R^{n}, R^{n}\right]$ possess mixed quasi monotone property, and let $v_{0}, w_{0}$ be coupled lower and upper quasi solutions of system (3.4) on $N_{0}^{+}$.Suppose further that

$$
f_{i}\left(n, u_{i},[u]_{p i},[u]_{q i}\right)-f_{i}\left(n, s_{i},[u]_{p i},[u]_{q i}\right) \geq-M\left(u_{i}-s_{i}\right)
$$


whenever $v_{0} \leq u \leq w_{0}$ and $v_{0, i} \leq s_{i} \leq u_{i} \leq w_{0, i}$ and $M>0$. Then there exists monotone sequences $\left\{v_{n}\right\},\left\{w_{n}\right\}$ such that $v_{n} \rightarrow v$ and $w_{n} \rightarrow w$ as $n \rightarrow \infty$ uniformly and monotonically to coupled minimal and maximal quasi solutions of (3.4)on $N_{0}^{+}$provided $v_{0}(0) \leq u_{0} \leq w_{0}(0)$. Further if $u$ is any solution of (3.4) such that $v_{0} \leq u \leq w_{0}$ on $N_{0}^{+}$then $v \leq u \leq w$ on $N_{0}^{+}$.

Proof. For any $y, z \in C\left[N_{0}^{+}, R^{n}\right]$ such that $v_{0} \leq y, z \leq w_{0}$ on $N_{0}^{+}$, we define

$$
\nabla^{\alpha} u_{i+1}=f_{i}\left(n, y_{i},[y]_{p i},[z]_{q i}\right)-M\left[u_{i}-y_{i}\right], u(0)=u_{0}
$$

Clearly, (3.7) is a non-homogeneous equation in $u_{i}$ and has an unique solution [8]. In order to construct and establish the convergence of the monotone sequences $\left\{v_{n}\right\},\left\{w_{n}\right\}$, we define a mapping $A$ such that $A[y, z]=u$, where $u$ is the unique solution of (3.7). This sequences $\left\{v_{n}\right\}$, are $\left\{w_{n}\right\}$ derived in the following steps.

(a). $v_{0} \leq A\left[v_{0}, w_{0}\right], w_{0} \geq A\left[w_{0}, v_{0}\right]$.

(b). 'A' possesses the mixed quasi monotone property on the segment $\left[v_{0}, w_{0}\right]$, where the segment $\left[v_{0}, w_{0}\right]=\left\{u \in C\left[N_{0}^{+}, R^{n}\right]: v_{0} \leq u \leq w_{0}\right\}$.

To prove (a): Let $v_{1}$ be the unique solution of (3.7) with $y=v_{0}, z=w_{0}$. Then $A\left[v_{0}, w_{0}\right]=v_{1}$. Now let $p_{i}=v_{0, i}-v_{1, i}$ and consider

$$
\begin{aligned}
& \nabla^{\alpha} p_{i+1}=\nabla^{\alpha}\left[v_{0, i+1}-v_{1, i+1}\right] \\
& \leq f_{i}\left(n, v_{0, i},\left[v_{0}\right]_{p i},\left[w_{0}\right]_{q i}\right) \\
& -f_{i}\left(n, v_{0, i},\left[v_{0}\right]_{p i},\left[w_{0}\right]_{q i}\right)+M\left[v_{1, i}-v_{0, i}\right] \\
& \leq-M p_{i} \text {. } \\
& \text { And also } p_{i}(0) \leq v_{0, i}(0)-v_{1, i}(0) \leq u(0)-u(0)=0 \text {. }
\end{aligned}
$$

Hence by Theorem 3.2, we have $p_{i} \leq 0$. Thus $v_{0, i} \leq v_{1, i}$, i.e., $v_{0, i} \leq A\left[v_{0, i}, w_{0, i}\right]$.

Similarly we can prove $w_{0, i} \geq A\left[w_{0, i}, v_{0, i}\right]$.

To prove (b): Let us take $y_{1}, y_{2}, z \in\left[v_{0}, w_{0}\right]$ be such that $y_{1} \leq y_{2}$. Suppose $A\left[y_{1}, z\right]=u_{1}$ and $A\left[y_{2}, z\right]=u_{2}$. Take $p_{i}=u_{1, i}-u_{2, i}$.

$$
\begin{aligned}
\nabla^{\alpha} p_{i+1}= & \nabla^{\alpha}\left[u_{1, i+1}-u_{2, i+1}\right] \\
= & f_{i}\left(n, y_{1, i},\left[y_{1}\right]_{p i},[z]_{q i}\right)-M\left[u_{1, i}-y_{1, i}\right]-f_{i}\left(n, y_{2, i},\left[y_{2}\right]_{p i},[z]_{q i}\right) \\
& +M\left[u_{2, i}-y_{2, i}\right] \\
\leq & M\left[y_{2, i}-y_{1, i}\right]-M\left[u_{1, i}-y_{1, i}\right]+M\left[u_{2, i}-y_{2, i}\right] \\
\leq & -M\left[u_{1, i}-u_{2, i}\right]
\end{aligned}
$$




$$
\leq-M p_{i}
$$

Using similar arguments as above, we have $p_{i+1} \leq 0$. Thus $u_{1, i} \leq u_{2, i}$ or $A\left[y_{1}, z\right] \leq A\left[y_{2}, z\right]$. Similarly if $y, z_{1}, z_{2}, \in\left[v_{0}, w_{0}\right]$ such that $z_{1} \leq z_{2}$, then as before we can prove that $A\left[y, z_{1}\right] \geq A\left[y, z_{2}\right]$.

Consequently this implies that $A[y, z] \leq A[z, y]$ for $y \leq z$. Or $A$ satisfies mixed quasi monotone property.

Continuing this way using (a) and (b) ,we can define the sequences

$$
v_{n}=A\left[v_{n-1}, w_{n-1}\right], \quad w_{n}=A\left[w_{n-1}, v_{n-1}\right]
$$

satisfying

$$
v_{0} \leq v_{1} \leq \ldots \leq v_{n} \leq w_{n} \leq \ldots \leq w_{1} \leq w_{0} .
$$

Since the sequences are monotonic and hence by Dini's theorem they converge uniformly to coupled quasi solutions of $(v, w)$ of $(3.7)$.

Therefore $v=\lim _{n \rightarrow \infty} v_{n}, w=\lim _{n \rightarrow \infty} w_{n}$. Hence $v_{i}(n), w_{i}(n)$ satisfy

$$
\begin{aligned}
\nabla^{\alpha} v_{i+1} & =f_{i}\left(n, v_{i},[v]_{p i},[w]_{q i}\right), v(0)=u_{0} . \\
\nabla^{\alpha} w_{i+1} & =f_{i}\left(n, v_{i},[w]_{p i},[v]_{q i}\right), w(0)=u_{0} .
\end{aligned}
$$

Now we show that $(v, w)$ are coupled minimal and maximal quasi solutions of (3.4) respectively. Let $\left(u_{1}, u_{2}\right)$ be any coupled quasi solutions of (3.4) such that $u_{1}, u_{2} \in\left[v_{0}, w_{0}\right]$.

Let us suppose that for some integer $k>0, v_{k} \leq u_{1}, u_{2} \leq w_{k}$ on $N_{0}^{+}$.

Now we take $p_{i}=v_{k+1, i}-u_{1, i}$ and using the mixed quasi monotone property of $f$ we have

$$
\begin{aligned}
\nabla^{\alpha} p_{i+1} & =\nabla^{\alpha}\left[v_{k+1, i+1}-u_{1, i+1}\right] \\
& =f_{i}\left(n, v_{k}(i),\left[u_{1}\right]_{p i},\left[u_{2}\right]_{q i}\right)-M\left(v_{k+1, i}-v_{k, i}\right)-f_{i}\left(n, u_{1, i},\left[u_{1}\right]_{p i},\left[u_{2}\right]_{q i}\right), \\
& \leq M\left(u_{1, i}-v_{k, i}\right)-M\left(v_{k+1, i}-v_{k, i}\right), \\
& \leq-M p_{i} .
\end{aligned}
$$

Also $p_{i}(0) \leq 0$ implies that $p_{i} \leq 0$. Thus $v_{k} \leq u_{1}$. Similarly we can prove $u_{1} \leq w_{k+1}$ also. By the principle of mathematical induction, it can be proved that, for every $m \in \mathbb{N}_{0}^{+}, v_{m}(n) \leq u_{1}(n), u_{2}(n) \leq w_{m}(n)$. As $m \rightarrow \infty$, we have $v(n) \leq u_{1}(n), u_{2}(n) \leq w(n)$. Hence the functions $v(n)$ and $w(n)$ defined on $n \in \mathbb{N}_{0}^{+}$are minimal and maximal quasi solutions of (3.4) respectively.

Since any solution $u \in\left[v_{0}, w_{0}\right]$ can be considered as $(u, u)$ coupled quasi solutions of (3.4), we also have $v \leq u \leq w$ on $n \in \mathbb{N}_{0}^{+}$.

This completes the proof. 


\section{References}

[1] Agarwal, R.P., Difference equations and inequalities, Marcel Dekker, New York, 1992.

[2] Deekshitulu, G.V.S.R. and Jagan Mohan, J., Fractional difference inequalities, Communications in Applied Analysis, 14, No. 1 (2010), 89-98.

[3] Deekshitulu, G.V.S.R. and Jagan Mohan, J., Fractional difference inequalities of Bihari type, Communications in Applied Analysis, 14, No. 4 (2010), 343-354.

[4] Deekshitulu, G.V.S.R. and Jagan Mohan, J., Fractional difference inequalities of Volterra type, International Journal of Pure and Applied Mathematics, 70, No. 2 (2011), 137-149.

[5] Deekshitulu, G.V.S.R. and Jagan Mohan, J., Fractional difference inequalities of Opial type and initial value problem, Fractional Differential Calculus, 2, No. 1 (2012), 73-86.

[6] Deekshitulu, G.V.S.R. and Jagan Mohan, J., Some New Fractional Difference Inequalities of Gronwall - Bellman Type, Mathematical Sciences, Springer Open, Volume 6, Number 69, doi: 10.1186/2251-7456-6-69.

[7] Diaz, J.B. and Osler, T.J., Differences of fractional order, Math. Comp., 28 (1974), 185-201.

[8] G.V.S.R. Deekshitulu and J.J Mohan, Solutions of perturbed nabla fractional difference equations of order $0<\alpha<1$, Mathematica Aeterna, 3, No. 2, 139-150.

[9] Henry L Gray and Nien fan Zhang, On a New Definition of the Fractional Difference, Math. Comp., 50 (1988), 513-529.

[10] Hirota, R., Lectures on difference equations, Science-sha, 2000, In Japanese.

[11] Nagai, A., An integrable mapping with fractional difference, J. Phys. Soc. Jpn., 72 (2003), 2181-2183.

[12] Pachpatte, B.G., Inequalities of finite difference equations, Marcel Dekker, New York, 2002.

[13] Podlubny, I, Fractional differential equations, Academic Press, San Diego, 1999. 